T. Ono

Nagoya Math. J.

Vol. 55 (1974), 145-150

\title{
ON SOME RATIONALITY PROPERTIES OF HOPF MAPS
}

\author{
TAKASHI ONO
}

As is well known, the Hopf fibration $S^{3} \rightarrow S^{2}$ is the restriction of the map $h: \boldsymbol{R}^{4} \rightarrow \boldsymbol{R}^{3}$ given by $h(x)=\left(x_{1}^{2}+x_{2}^{2}-x_{3}^{2}-x_{4}^{2}, 2\left(x_{1} x_{3}-x_{2} x_{4}\right), 2\left(x_{1} x_{4}+\right.\right.$ $\left.x_{2} x_{3}\right)$ ), a system of three quadratic forms [2]. Since spheres and the map are defined by polynomials with coefficients in $Q$, the original setting can be considered as a localization at infinity of the underlying algebraic sets and morphism defined over $\boldsymbol{Q}$. This makes one think of the arithmetic of the Hopf maps.

The purpose of this note is to arrange some basic materials so that one can see the algebraic mechanism of the Hopf's construction of quadratic maps mapping sphere to sphere and to prove a single Theorem concerning the surjectivity of the Hopf maps defined over an algebraic number field.

We shall use freely the results on a single quadratic form in [4]. As for elementary properties of the Clifford algebra, see [1].

\section{§ 1. Involutorial representation of Clifford algebras}

Let $K$ be a field of characteristic not two and $(V, q)$ be a nondegenerate quadratic space over $K$. We shall denote by $C(q)$ the Clifford algebra of $(V, q)$. We consider $V$, as usual, as a subspace of $C(q)$. The algebra $C(q)$ has a unique involution $c \mapsto \bar{c}$ such that $\bar{v}=-v$ for all $v \in V$.

Let $(Y, \varphi)$ be another non-degenerate quadratic space over $K$. We denote by $\Phi$ the symmetric bilinear form on $Y$ associated to $\varphi$, i.e. $\Phi\left(y_{1}, y_{2}\right)=\frac{1}{2}\left(\varphi\left(y_{1}+y_{2}\right)-\varphi\left(y_{1}\right)-\varphi\left(y_{2}\right)\right), y_{1}, y_{2} \in Y$. The quadratic form $\varphi$ defines an involution $a \mapsto a^{*}$ of the algebra $E=$ End $Y$ of $K$-endomorphisms of the space $Y$ by the rule

$$
\Phi\left(a y_{1}, y_{2}\right)=\Phi\left(y_{1}, a^{*} y_{2}\right), \quad a \in E .
$$

Received January 28, 1974. 
By an involutorial representation of $C(q)$ on $(Y, \varphi)$ we shall mean a homomorphism $\rho: C(q) \rightarrow E$ such that $\rho\left(e_{0}\right)=1_{E}, e_{0}$ being the identity of $C(q)$, and that $\rho(\bar{c})=\rho(c)^{*}$ for all $c \in C(q)$.

Put $X=K e_{0} \oplus V$ and $\theta(x)=x_{0}^{2}-q(v)$ for $x=x_{0} e_{0}+v, x_{0} \in K, v \in V$. We thus obtain another non-degenerate quadratic space $(X, \theta)$ over $K$. The following is a basic property of an involutorial representation $\rho$ :

$$
\rho(x)^{*} \rho(x)=\theta(x) 1_{E}, \quad x \in X .
$$

In fact, since $\rho(x)=x_{0} 1_{E}+\rho(v)$, we have $\rho(x)^{*}=x_{0} 1_{E}+\rho(v)^{*}=x_{0} 1_{E}$ $-\rho(v)$. Therefore we have $\rho(x)^{*} \rho(x)=x_{0}^{2} 1_{E}-\rho(v)^{2}=x_{0}^{2} 1_{E}-\rho\left(q(v) e_{0}\right)=$ $\theta(x) 1_{E}$, q.e.d.

From (1.2) we get

$$
(\operatorname{det} \rho(x))^{2}=\theta(x)^{n}, \quad x \in X, n=\operatorname{dim} Y \text {. }
$$

From (1.1), (1.2) we get

$$
\varphi(\rho(x) y)=\theta(x) \varphi(y), \quad x \in X, y \in Y .
$$

This relation implies that $\operatorname{dim} X \leqq \operatorname{dim} Y$.

To each involutorial representation $\rho$ one associates the Hopf map $h_{\rho}: X \oplus Y \rightarrow K \oplus Y$ by

$$
h_{\rho}(x, y)=(\theta(x)-\varphi(y), 2 \rho(x) y) .
$$

Using (1.4) we observe that $(\theta(x)-\varphi(y))^{2}+\varphi(2 \rho(x) y)=(\theta(x)+\varphi(y))^{2}$. Hence, if we put

$$
f(x, y)=\theta(x)+\varphi(y), \quad g(u, y)=u^{2}+\varphi(y), \quad x \in X, y \in Y, u \in K,
$$

we will obtain non-degenerate quadratic space $(X \oplus Y, f)$ and $(K \oplus Y, g)$, and the relation

$$
g\left(h_{\rho}(z)\right)=f(z)^{2}, \quad z=(x, y) \in X \oplus Y .
$$

In other words, if we put, for $t \in K$,

$$
S_{f}(t)=\{z \in X \oplus Y, f(z)=t\}, \quad S_{g}(t)=\{w \in K \oplus Y, g(w)=t\},
$$

then the map $h_{\rho}$ induces a map $h_{\rho}(t): S_{f}(t) \rightarrow S_{g}\left(t^{2}\right)$ of two "spheres".

Remark (1.5). The orthogonal group $O(\varphi)$ acts on involutorial representations by $\rho^{\sigma}(c)=\sigma \rho(c) \sigma^{-1}, \sigma \in O(\varphi), c \in C(q)$. One then verifies easily that $h_{\rho} \sigma_{1}=\sigma_{2} h_{\rho}$ where $\sigma_{1}(x, y)=(x, \sigma y), \sigma_{2}(u, y)=(u, \sigma y)$ for $\sigma \in O(\varphi)$. 


\section{§ 2. Regular representation of Clifford algebras}

Here we shall show that any non-degenerate quadratic space $(V, q)$ gives rise to a Hopf map.

For this purpose we shall take as $\rho$ the regular representation $C(q)$ $\rightarrow E=$ End $C(q)$ and find a quadratic from $\varphi$ on the vector space $C(q)$ so that $\rho$ becomes an involutorial representation of $C(q)$ on the quadratic space $(C(q), \varphi)$.

Let $\left\{e_{i}\right\}, 1 \leqq i \leqq m$, be an orthogonal base of $(V, q)$ and put $a_{i}=q\left(e_{i}\right)$. To each subset $A$ of $M=\{1,2, \cdots, m\}$ we put $e_{A}=e_{i_{1}} \cdots e_{i_{\alpha}}, a_{A}=a_{i_{1}} \cdots$ $a_{i_{\alpha}}$ when $A=\left\{i_{1}, \cdots, i_{\alpha}\right\}$ with $i_{1}<\cdots<i_{\alpha}$, and put $e_{\phi}=e_{0}$. For two subsets $A$ and $B$ of $M$, we denote by $A+B$ the set $(A \cup B)-(A \cap B)$. This operation has the properties $A+(B+C)=(A+B)+C, A+A$ $=\phi$ and $A+\phi=A$. It is well known that

$$
e_{A} e_{B}=\gamma_{A, B} e_{A+B}
$$

with

$$
\gamma_{A, B}=\prod_{a, b}(a, b) \prod_{i \in A \cap B} a_{i}, \quad a \in A, b \in B,
$$

where $(a, b)=1$ if $a \leqq b$ and -1 if $a>b$.

Now, let $\rho(x)=\left(R_{C B}(x)\right), x \in C(q)$, be the regular representation of $C(q)$ with respect to the base $\left\{e_{A}\right\}: e_{A} e_{B}=\sum_{C} R_{C B}\left(e_{A}\right) e_{C}$. We have then $R_{C B}\left(e_{A}\right)=\gamma_{A, B}$ if $C=A+B$, =0 if $C \neq A+B$.

Next, let $\left\{E_{A B}\right\}$ be the standard base of the total matric algebra $E=$ End $C(q)$ with respect to the base $\left\{e_{A}\right\}$ and put $F=\sum_{A}(-1)^{\alpha} a_{A} E_{A A}$, this being the diagonal matrix in $E$ whose diagonal elements are $(-1)^{\alpha} a_{A}$. Identifying $y=\sum_{A} y_{A} e_{A} \in C(q)$ with the vertical vector $\left(y_{A}\right)$, we shall show that the quadratic form $\varphi(y)={ }^{t} y F y$ is the one we are looking for. First note that $a^{*}=F^{-1 t} a F, a \in E$, when $a$ is considered as a matrix with respect to the base $\left\{E_{A B}\right\}$. Since $\rho$ is a homomorphism, to see that $\rho$ is involutorial on $(C(q), \varphi)$, it is enough to show that

$$
F \rho\left(e_{i}\right)+{ }^{t} \rho\left(e_{i}\right) F=0, \quad 1 \leqq i \leqq m,
$$

or, equivalently, that

$$
(-1)^{\alpha} a_{A} R_{A B}\left(e_{i}\right)+(-1)^{\beta} a_{B} R_{B A}\left(e_{i}\right)=0, \quad 1 \leqq i \leqq m .
$$

Now, if $A \neq B+\{i\}$ and $B \neq A+\{i\}$, then $R_{A B}\left(e_{i}\right)=R_{B A}\left(e_{i}\right)=0$; otherwise, one must have $A=B+\{i\}$ and $B=A+\{i\}$ because we have, in 
general, $C=A+B$ if and only if $B=A+C$ as is seen from the properties of $A+B$ mentioned above. Hence, we are reduced to verify the following:

$$
(-1)^{\alpha} a_{A} \gamma_{\{i\}, A+\{i\}}+(-1)^{\alpha+1} a_{A+(i\}} \gamma_{(i), A}=0, \quad 1 \leqq i \leqq m .
$$

Actually, we only have to consider the case where $i \notin A$ because if $i \in A$ then $i \notin A+\{i\}$ and so we can use $A+\{i\}$ in place of $A=(A+\{i\})+\{i\}$. Then (2.1) follows from the relation:

$$
\gamma_{\{i\}, A+\{i\}}=\prod_{a \in A+\{i\}}(i, a) a_{i}=\gamma_{\{i\}, A} a_{i}
$$

Remark (2.2). If $K=\boldsymbol{R}$ and $q$ is negative definite, then all $a_{i}<0$ and hence all $(-1)^{\alpha} a_{A}>0$, i.e. $\varphi$ is positive definite.

Remark (2.3). For any $K$, if all $a_{i}=-1$, then all $(-1)^{\alpha} a_{A}=1$. In this case, all quadratic forms $\theta, \varphi, f, g$ are of standard type, i.e. the sum of squares. Our previous paper [3] treated this case only.

\section{§ 3. Hopf maps over number fields}

Let $k$ be an algebraic number field of finite degree over the field $\boldsymbol{Q}$ of rationals. For a place $v$ of $k$ we denote by $k_{v}$ the completion of $k$ at $v$. An element $a \in k$ is called totally positive if $a$ is positive in $k_{v}$ for all real infinite place $v$.

Let $(V, q)$ be a non-degenerate quadratic space over $k$. For a place $v$, we denote by $\left(V_{v}, q_{v}\right)$ the quadratic space over $k_{v}$ obtained from $(V, q)$ by the field extension. We denote by $M(q)$ the set of all real places $v$ for which $q_{v}$ is negative definite and make the assumption that $M(q)$ is not empty.

Let $(Y, \varphi)$ be a non-degenerate quadratic space over $k$ such that $\varphi_{v}$ is positive definite for all $v \in M(q)$ and let $\rho$ be an involutorial representation of $C(q)$ on $(Y, \varphi)$. Let $t$ be an element of $k$ such that $t$ is positive in $k_{v}$ for all $v \in M(q)$. As described in $\S 1$, the Hopf map $h_{\rho}: X \oplus Y$ $\rightarrow k \oplus Y$ induces a map $h_{\rho}(t): S_{f}(t) \rightarrow S_{g}\left(t^{2}\right)$.

THEOREM. Notation and assumptions being as above, the map $h_{\rho}(t)$ is surjective whenever $\operatorname{dim} V \geqq 3$.

Proof. Take any point $\gamma=(\alpha, \beta) \in S_{g}\left(t^{2}\right) \subset k \oplus Y$. We wish to find 
$\zeta=(\xi, \eta) \in S_{f}(t) \subset X \oplus Y$ such that $h_{\rho}(\zeta)=\gamma$. In other words, under the assumption

$$
\alpha^{2}+\varphi(\beta)=t^{2}, \quad \alpha \in k, \beta \in Y,
$$

we must find $(\xi, \eta) \in X \oplus Y$ such that

$$
\theta(\xi)+\varphi(\eta)=t, \quad \theta(\xi)-\varphi(\eta)=\alpha, \quad 2 \rho(\xi) \eta=\beta .
$$

If we put $a=\frac{1}{2}(t+\alpha), b=\frac{1}{2}(t-\alpha)$, the problem is to solve the following system of equations

$$
\begin{gathered}
\theta(\xi)=a \\
\varphi(\eta)=b \\
2 \rho(\xi) \eta=\beta
\end{gathered}
$$

under the assumption

$$
\varphi(\beta)=4 a b .
$$

We shall split our argument into two cases.

Case 1. $a \neq 0$.

In this case, since $\operatorname{dim} X=\operatorname{dim} V+1 \geqq 4$, the equations (3.1) has a solution $\xi_{v} \in X_{v}$ for each finite place $v$. As for the infinite place $v$, if $v$ is complex (3.1) has obviously a solution $\xi_{v}$; the same is true if $v$ is real for which $\theta_{v}$ is indefinite. So suppose that $v$ is real and $\theta_{v}$ is definite. Then, since $\theta_{v}=x_{0}^{2}-q_{v}, \theta_{v}$ must be positive definite and $q_{v}$ must be negative definite, and hence $v \in M(q)$. Hence, by the assumption, $\varphi_{v}$ is positive definite and $t>0$ in $k_{v}$. Then, the condition (3.4) implies that $a>0$ in $k_{v}$ because otherwise we have $a<0, b \leqq 0$ in $k_{v}$, and $t=a+b<0$ in $k_{v}$, a contradiction. Thus, (3.1) has again a solution for such a real place $v$. Therefore, by Hasse principle for $\theta$, (3.1) has a rational solution $\xi \in X$. Since $a \neq 0$, it follows from (1.3) that $\rho(\xi)$ is invertible, and so we are forced to set $\eta=\frac{1}{2} \rho(\xi)^{-1} \beta$ and then one sees easily that this $\eta$ automatically satisfies (3.2) by virtue of (1.4), (3.1) and (3.4).

Case 2. $a=0$.

In this case, (3.4) becomes $\varphi(\beta)=0$. Take a real place $v$ in $M(q)$. Then $\varphi_{v}(\beta)=\varphi(\beta)=0$ implies that $\beta=0$ by the assumption on $\varphi_{v}$. As $\xi=0$ and any $\eta$ satisfies (3.1) and (3.3), we only have to solve (3.2). Now, since $\operatorname{dim} Y \geqq \operatorname{dim} X \geqq 4$, we have local solutions of (3.2) for all 
finite and infinite places except possibly for real $v$ for which $\varphi_{v}$ is definite. But for such $v$, it follows from (1.4) that $\theta_{v}$ is definite and, by the same argument as in Case 1, we see that $v \in M(q)$. Since $b=t$ in this case, (3.2) has a solution for $v$ and, by Hasse principle, it has a rational solution $\eta \in Y$, q.e.d.

Remark (3.5). From (2.2), we see that the space $(Y, \varphi)$ associated to $(V, q)$ by using the regular representation in $\S 2$ satisfies the assumption of the Theorem.

Remark (3.6). Consider the case $k=Q$ and $q(v)=-\left(x_{1}^{2}+\cdots+x_{m}^{2}\right)$. If we apply the construction of $\S 2$ to $(V, q), V=Q^{m}$, we obtain the space $(Y, \varphi)$ with $\varphi(y)=y_{1}^{2}+\cdots+y_{n}^{2}, n=2^{m}, y \in Y=C(q)=Q^{n}$. We also get

$$
\begin{aligned}
& f(x, y)=x_{0}^{2}+x_{1}^{2}+\cdots+x_{m}^{2}+y_{1}^{2}+\cdots+y_{n}^{2}, \\
& g(u, y)=u^{2}+y_{1}^{2}+\cdots+y_{n}^{2} .
\end{aligned}
$$

For $t>0, t \in Q$, the map $h_{\rho}(t) \operatorname{maps} S_{f}(t)\left(\left(m+2^{m}\right)\right.$-sphere over $\left.Q\right)$ onto $S_{g}\left(t^{2}\right) \quad\left(2^{m}\right.$-sphere over $Q$ ) provided $m \geqq 3$ (Theorem). Let us examine the case $m=1$. In this case, the localization of $h_{\rho}(1)$ at $v=\infty$ is the classical Hopf fibration: $S^{3} \rightarrow S^{2}$ which is onto, of course. However, the global map $h_{\rho}(1)$ is not onto. For example, take $\gamma=(\alpha, \beta) \in \boldsymbol{Q} \oplus Y=\boldsymbol{Q}^{3}$ with $\alpha=1 / 3, \beta=(2 / 3,2 / 3)$. Clearly $\gamma \in S_{g}(1)$. If there were $\zeta=(\xi, \eta) \in S_{f}(1)$ such that $h_{\rho}(\zeta)=\gamma$, then one would have $\theta(\xi)+\varphi(\eta)=\xi_{0}^{2}+\xi_{1}^{2}+\eta_{1}^{2}+\eta_{2}^{2}=$ $1, \theta(\xi)-\varphi(\eta)=\xi_{0}^{2}+\xi_{1}^{2}-\eta_{1}^{2}-\eta_{2}^{2}=\alpha=1 / 3$, and so $\xi_{0}^{2}+\xi_{1}^{2}=2 / 3$; but this is impossible because the Hilbert symbol $(6,6)_{3}=(-1 / 3)=-1$.

\section{REFERENCES}

[1] E. Artin, Geometric Algebra, Interscience Publishers, New York, 1957.

[2] H. Hopf, Über die Abbildungen der dreidimensionalen Sphäre auf die Kugelfläche, Math. Ann. 104 (1931), 637-665.

[ 3 ] T. Ono, Hasse Principle for Hopf Maps (to appear).

[ 4 ] E. Witt, Theorie der quadratischen Formen in beliebigen Körpern, J. reine angew. Math. 176 (1937), 31-44.

The Johns Hopkins University 